\title{
QUANTUM LEEVY-TYPE LAPLACIAN AND ASSOCIATED STOCHASTIC DIFFERENTIAL EQUATIONS
}

\author{
A. BARHOUMI and H. OUERDIANE \\ Department of Mathematics \\ Faculty of Sciences of Tunis \\ University of Tunis El-Manar \\ 1060 Tunis, Tunisia \\ E-mail: abdessatar.barhoumi@ipein.rnu.tn,habib.ouerdiane@fst.rnu.tn
}

\begin{abstract}
We study a quantum extension of the Lévy Laplacian, so-called quantum Lévy-type Laplacian, to the nuclear algebra of operators on spaces of entire functions. We give several examples of the action of the quantum Lévy-type Laplacian on basic operators and we study a quantum white noise convolution differential equation involving the quantum Lévy-type Laplacian.
\end{abstract}

1. Introduction. In infinite dimensional analysis, the Lévy Laplacian was introduced by P. Lévy [19] and rigorously developed by T. Hida and his school [14], [25], [22], [18].

The situation however changed with a series of papers [3], [4] where it is proved that the Yang-Mills equations and the Lévy Laplace equation for the associated parallel transport are equivalent. These results provide a strong motivation for a new approach to infinite dimensional analysis based on the Brownian motion and its quantization. On the other hand, a Fock space realization of the Lévy Brownian motion is obtained and the corresponding quantum process is given explicitly [2]. More recently, an infinite dimensional classical stochastic process generated by self-adjoint extensions of the Lévy Laplacian is discussed and equi-continuous semigroups of class $\left(C_{0}\right)$ generated by these extensions are constructed [25].

The present work is mostly based on the papers [1], [24], [22] where a first path to a quantum approach to the Lévy Laplacian is done. Then, employing the recent framework of the theory of operators defined on spaces of holomorphic functions [7], the convolution calculus [6], and the heat equation associated with the Lévy Laplacian [22], [24], [4], we

2000 Mathematics Subject Classification: Primary 60H40; Secondary 46A32, 46F25, 46G20.

Key words and phrases: convolution product, Gelfand triple, heat equation, holomorphy, Laplace transform, Lévy Laplacian, operator symbol, quadratic quantum white noise.

The paper is in final form and no version of it will be published elsewhere. 
specify the action of the quantum Lévy-type Laplacian on basic examples of operators and we give the operator version of the nice connection between the Lévy Laplacian and the quadratic quantum white noise pointed out, in the classical case, in [22], [24].

The paper is organized as follows. In Section 2 we assemble a general framework which is necessary for our paper. In Section 3 we reformulate the so-called quantum Lévy-type Laplacian. In Section 4 we give several examples of the action on basic white noise operators and we show, in particular, that in the case of the commutative subalgebra of convolution operators, the classical Lévy Laplacian coincides with its quantum extension via the Laplace transform and the symbol transform. Section 5 is devoted to an investigation of a quantum white noise differential equation involving the quantum Lévy-type Laplacian. Finally, we discuss the traditional dependence of infinite dimensional Laplacians and some infinite sequences of vectors.

General notation. Let $\mathcal{X}, \mathcal{Y}$ be locally convex spaces.

$\triangleright \mathcal{L}(\mathcal{X}, \mathcal{Y})$ : the space of all continuous linear operators from $\mathcal{X}$ into $\mathcal{Y}$ equipped with the topology of bounded convergence.

$\triangleright \mathcal{X} \otimes \mathcal{Y}:$ the complete Hilbert space tensor product when both $\mathcal{X}, \mathcal{Y}$ are Hilbert spaces. $\triangleright \mathcal{X} \otimes_{\pi} \mathcal{Y}:$ the complete $\pi$-tensor product. For simplicity, with no danger of confusion in our context, $\otimes_{\pi}$ will be denoted by $\otimes$ again.

$\triangleright$ In both cases, $\hat{\otimes}$ stands for the symmetric tensor product.

2. General framework. First we review basic concepts, notations, and some results which will be needed in the present paper. Development of these and similar results can be found in the papers [12], [23], [6], [7].

2.1. Entire function with $\theta$-exponential growth. Let $\theta$ be a Young function, i.e., it is a continuous, convex and increasing function defined on $\mathbb{R}_{+}$and satisfies the two conditions: $\theta(0)=0$ and $\lim _{x \rightarrow \infty} \theta(x) / x=+\infty$, see [11]. We define the conjugate function $\theta^{*}$ of $\theta$ by

$$
\theta^{*}(x)=\sup _{t \geq 0}(t x-\theta(t)), \quad x \geq 0 .
$$

Throughout the paper, we fix a Young function $\theta$.

For a complex Banach space $(B,\|\|$.$) let \mathcal{H}(B)$ denote the space of all entire functions on $B$, i.e. of all continuous $\mathbb{C}$-valued function on $B$ whose restrictions to all affine lines of $B$ are entire on $\mathbb{C}$. For each $m>0$ we denote by $\operatorname{Exp}(B, \theta, m)$ the space of all entire functions on $B$ with $\theta$-exponential growth of finite type $m$, i.e.

$$
\operatorname{Exp}(B, \theta, m)=\left\{f \in \mathcal{H}(B) ;\|f\|_{\theta, m}:=\sup _{z \in B}|f(z)| e^{-\theta(m\|z\|)}<\infty\right\} .
$$

Let $E$ be a real nuclear Fréchet space with topology given by an increasing family $\left\{|\cdot|_{p} ; p \in \mathbb{N}\right\}$ of Hilbertian norms. Then

$$
E=\underset{p \rightarrow \infty}{\operatorname{proj} \lim } E_{p},
$$

where $E_{p}$ is the completion of $E$ with respect to the norm $|\cdot|_{p}$. We use $E_{-p}$ to denote the topological dual space of $E_{p}$. Then the strong dual space $E^{\prime}$ can be obtained as

$$
E^{\prime}=\operatorname{indlim}_{p \rightarrow \infty} E_{-p}
$$


where the strong topology of $E^{\prime}$ and the inductive limit topology coincide due to the nuclearity of $E$.

Let $N=E+i E$ and $N_{p}=E_{p}+i E_{p}, p \in \mathbb{Z}$ be the complexifications of $E$ and $E_{p}$, respectively. Then $N$ and its strong dual space $N^{\prime}$ can be represented by

$$
N=\underset{p \rightarrow \infty}{\operatorname{projlim}} N_{p} \text { and } N^{\prime}=\underset{p \rightarrow \infty}{\operatorname{indim}} N_{-p}
$$

According to (2.1), the projective system $\left\{\operatorname{Exp}\left(N_{-p}, \theta, m\right) ; p \in \mathbb{N}, m>0\right\}$ and the inductive system $\left\{\operatorname{Exp}\left(N_{p}, \theta, m\right) ; p \in \mathbb{N}, m>0\right\}$ give the following two nuclear spaces:

$$
\mathcal{F}_{\theta}\left(N^{\prime}\right)=\underset{p \rightarrow \infty ; m \downarrow 0}{\operatorname{projlim}} \operatorname{Exp}\left(N_{-p}, \theta, m\right), \quad \mathcal{G}_{\theta}(N)=\operatorname{indlim}_{p \rightarrow \infty ; m \rightarrow \infty} \operatorname{Exp}\left(N_{p}, \theta, m\right) .
$$

By definition $f \in \mathcal{F}_{\theta}\left(N^{\prime}\right)$ and $g \in \mathcal{G}_{\theta}(N)$ admit the Taylor expansions:

$$
\begin{gathered}
f(z)=\sum_{n=0}^{\infty}\left\langle z^{\otimes}, f_{n}\right\rangle, \quad z \in N^{\prime}, f_{n} \in N^{\hat{\otimes} n}, \\
g(\xi)=\sum_{n=0}^{\infty}\left\langle g_{n}, \xi^{\otimes n}\right\rangle, \quad \xi \in N, g_{n} \in\left(N^{\hat{\otimes} n}\right)^{\prime},
\end{gathered}
$$

where we used the common symbol $\langle\cdot, \cdot\rangle$ for the canonical $\mathbb{C}$-bilinear form on $\left(N^{\hat{\otimes} n}\right)^{\prime} \times N^{\hat{\otimes} n}$ for all $n$. The Taylor series map $\mathcal{T}$ (at zero) associates to any entire function the sequence of coefficients. For example, if $f \in \mathcal{F}_{\theta}\left(N^{\prime}\right)$ is given as in (2.2), the Taylor series map is defined by $\mathcal{T} f=\vec{f}=\left(f_{n}\right)$.

The spaces $\mathcal{F}_{\theta}\left(N^{\prime}\right)$ and $\mathcal{G}_{\theta}(N)$ are characterized through the $\mathcal{T}$-transform and the following two nuclear Fréchet spaces:

$$
F_{\theta}(N)=\underset{p \rightarrow \infty ; m \downarrow 0}{\operatorname{projlim}} F_{\theta, m}\left(N_{p}\right), \quad G_{\theta}\left(N^{\prime}\right)=\operatorname{indlim}_{p \rightarrow \infty ; m \rightarrow \infty} G_{\theta, m}\left(N_{-p}\right),
$$

where, for any pair $p \in \mathbb{N}, m>0$,

$$
\begin{gathered}
F_{\theta, m}\left(N_{p}\right)=\left\{\vec{f}=\left(f_{n}\right) ; f_{n} \in N_{p}^{\hat{\otimes} n},\|\vec{f}\|_{\theta, p, m}^{2}:=\sum_{n=0}^{\infty} \theta_{n}^{-2} m^{-n}\left|f_{n}\right|_{p}^{2}<\infty\right\}, \\
G_{\theta, m}\left(N_{-p}\right)=\left\{\vec{\Phi}=\left(\Phi_{n}\right) ; \Phi_{n} \in N_{-p}^{\hat{\otimes} n},\|\vec{\Phi}\|_{\theta,-p, m}^{2}:=\sum_{n=0}^{\infty}\left(n ! \theta_{n}\right)^{2} m^{n}\left|\Phi_{n}\right|_{-p}^{2}<\infty\right\}
\end{gathered}
$$

and

$$
\theta_{n}=\inf _{r>0} \frac{\exp (\theta(r))}{r^{n}}, \quad n=0,1,2, \ldots
$$

By definition, the strong dual of $F_{\theta}(N)$ is identified with $G_{\theta}\left(N^{\prime}\right)$ through the canonical C-bilinear form

$$
\langle\langle\vec{\Phi}, \vec{f}\rangle\rangle:=\sum_{n=0}^{\infty} n !\left\langle\Phi_{n}, f_{n}\right\rangle .
$$

Moreover, we have the following duality theorem:

TheOREM 2.1 ([12]). The Taylor series map $\mathcal{T}$ gives two topological isomorphisms

$$
\mathcal{F}_{\theta}\left(N^{\prime}\right) \rightarrow F_{\theta}(N) \quad \text { and } \quad \mathcal{G}_{\theta^{*}}(N) \rightarrow G_{\theta}\left(N^{\prime}\right) .
$$


Let $\mathcal{F}_{\theta}\left(N^{\prime}\right)^{*}$ denote the topological dual space of $\mathcal{F}_{\theta}\left(N^{\prime}\right)$. The action of a distribution $\Phi \in \mathcal{F}_{\theta}\left(N^{\prime}\right)^{*}$ on a test function $\varphi \in \mathcal{F}_{\theta}\left(N^{\prime}\right)$ can be expressed in terms of the Taylor map as follows:

$$
\langle\langle\Phi, \varphi\rangle\rangle=\langle\langle\vec{\Phi}, \vec{\varphi}\rangle\rangle,
$$

where $\vec{\Phi}=\left(\mathcal{T}^{*}\right)^{-1} \Phi$ and $\vec{\varphi}=\mathcal{T} \varphi$. On the other hand, it is easy to see that for each $\xi \in N$, the exponential function

$$
e_{\xi}(z):=e^{\langle z, \xi\rangle}, \quad z \in N^{\prime}
$$

lies in $\mathcal{F}_{\theta}\left(N^{\prime}\right)$ and the set of such test functions spans a dense subspace of $\mathcal{F}_{\theta}\left(N^{\prime}\right)$. Thus, for any $\Phi \in \mathcal{F}_{\theta}\left(N^{\prime}\right)^{*}$, the Laplace transform of $\Phi$ is defined by

$$
(\mathcal{L} \Phi)(\xi):=\left\langle\left\langle\Phi, e_{\xi}\right\rangle\right\rangle, \quad \xi \in N
$$

Therefore, we have the following duality theorem:

THEOREM 2.2 ([12]). The Laplace transform realizes a topological isomorphism

$$
\mathcal{L}: \mathcal{F}_{\theta}\left(N^{\prime}\right)^{*} \rightarrow \mathcal{G}_{\theta^{*}}(N)
$$

2.2. Entire functions in two variables. Let $M$ and $N$ be two nuclear Fréchet spaces with defining Hilbertian norms $\left\{|\cdot|_{M, p}\right\}$ and $\left\{|\cdot|_{N, p}\right\}$, respectively. Let $M_{p} \oplus N_{p}$ be the direct sum of Hilbert spaces. Then the direct sum $M \oplus N$ is by definition

$$
M \oplus N=\underset{p \rightarrow \infty}{\operatorname{projlim}} M_{p} \oplus N_{p}
$$

Similarly,

$$
(M \oplus N)^{\prime}=M^{\prime} \oplus N^{\prime}=\operatorname{indlim}_{p \rightarrow \infty} M_{-p} \oplus N_{-p} .
$$

By definition, an entire function in two variables on $M \times N$ is a separately entire function $f: M \times N \rightarrow \mathbb{C}$. On the other hand, in an obvious manner, a function $f: M \times N \rightarrow \mathbb{C}$ is in one-to-one correspondence to a function $\tilde{f}: M \oplus N \rightarrow \mathbb{C}$. Therefore, we will not make distinction between them. Fore more details see e.g. [11].

According to the previous subsection, we easily check that a function $f:(M \oplus N)^{\prime} \rightarrow \mathbb{C}$ belongs to $\mathcal{F}_{\theta}\left((M \oplus N)^{\prime}\right)$ if and only if for any pair $p \geq 0$ and $m>0$

$$
\|f\|_{\theta,-p, m}:=\sup _{\omega \in M^{\prime}, z \in N^{\prime}}|f(\omega \oplus z)| \exp \left(-\theta\left(m|\omega|_{M,-p}\right)-\theta\left(m|z|_{N,-p}\right)\right)<\infty .
$$

Similarly, a function $g: M \oplus N \rightarrow \mathbb{C}$ belongs to $\mathcal{G}_{\theta}(M \oplus N)$ if and only if there exists a pair $p \geq 0$ and $m>0$ such that

$$
\|g\|_{\theta, p, m}:=\sup _{\xi \in M, \eta \in N}|g(\xi \oplus \eta)| \exp \left(-\theta\left(m|\xi|_{M, p}\right)-\theta\left(m|\eta|_{N, p}\right)\right)<\infty .
$$

As in (2.3), an exponential function in two variables is defined, for $\xi \in M$ and $\eta \in N$, by

$$
e_{\xi \otimes \eta}(z, w)=\exp \{\langle z, \xi\rangle+\langle w, \eta\rangle\}, \quad z \in N^{\prime}, w \in M^{\prime}
$$

Then, the Laplace transform, denoted again by $\mathcal{L}$, is defined as in (2.4) and the statement in Theorem 2.2 remains valid. Moreover, by a standard argument with the Taylor 
expansion, we see that the correspondence $e_{\xi \oplus \eta} \leftrightarrow e_{\xi} \otimes e_{\eta}$ can be uniquely extended to an isomorphism

$$
\mathcal{F}_{\theta}\left((M \oplus N)^{\prime}\right) \cong \mathcal{F}_{\theta}\left(M^{\prime}\right) \otimes \mathcal{F}_{\theta}\left(N^{\prime}\right)
$$

2.3. Characterization of operators. We are interested in continuous operators from $\mathcal{F}_{\theta}\left(N^{\prime}\right)$ into $\mathcal{F}_{\theta}\left(N^{\prime}\right)^{*}$. The space of such operators is denoted by $\mathcal{L}\left(\mathcal{F}_{\theta}\left(N^{\prime}\right), \mathcal{F}_{\theta}\left(N^{\prime}\right)^{*}\right)$ and assumed to carry the bounded convergence topology.

Let $\mu$ be the standard Gaussian measure on $E^{\prime}$ uniquely specified by its characteristic function

$$
e^{-\frac{1}{2}|\xi|_{0}^{2}}=\int_{E^{\prime}} e^{i\langle x, \xi\rangle} \mu(d x), \quad \xi \in E,
$$

and denote by $\mathbb{H}$ the Hilbert space $L^{2}\left(E^{\prime}, \mu, \mathbb{C}\right)$. Moreover, we assume that the Young function $\theta$ satisfies the following condition:

$$
\limsup _{x \rightarrow \infty} \frac{\theta(x)}{x^{2}}<+\infty
$$

or equivalently

$$
\liminf _{x \rightarrow \infty} \frac{\theta^{*}(x)}{x^{2}}>0
$$

Then, under this condition on $\theta$, we see that the spaces $\mathcal{L}\left(\mathcal{F}_{\theta}\left(N^{\prime}\right), \mathcal{F}_{\theta}\left(N^{\prime}\right)\right)$ and $\mathcal{L}(\mathbb{H}, \mathbb{H})$ can be considered as subspaces of $\mathcal{L}\left(\mathcal{F}_{\theta}\left(N^{\prime}\right), \mathcal{F}_{\theta}\left(N^{\prime}\right)^{*}\right)$. Moreover, identified with its restriction to $\mathcal{F}_{\theta}\left(N^{\prime}\right)$, each operator $T \in \mathcal{L}\left(\mathcal{F}_{\theta}\left(N^{\prime}\right)^{*}, \mathcal{F}_{\theta}\left(N^{\prime}\right)^{*}\right)$ will be considered as an element of $\mathcal{L}\left(\mathcal{F}_{\theta}\left(N^{\prime}\right), \mathcal{F}_{\theta}\left(N^{\prime}\right)^{*}\right)$, so that we have the continuous inclusion

$$
\mathcal{L}\left(\mathcal{F}_{\theta}\left(N^{\prime}\right)^{*}, \mathcal{F}_{\theta}\left(N^{\prime}\right)^{*}\right) \subset \mathcal{L}\left(\mathcal{F}_{\theta}\left(N^{\prime}\right), \mathcal{F}_{\theta}\left(N^{\prime}\right)^{*}\right) .
$$

In view of the classical kernel theorem, there is an isomorphism

$$
\mathcal{L}\left(\mathcal{F}_{\theta}\left(N^{\prime}\right), \mathcal{F}_{\theta}\left(N^{\prime}\right)^{*}\right) \cong \mathcal{F}_{\theta}\left(N^{\prime}\right)^{*} \otimes \mathcal{F}_{\theta}\left(N^{\prime}\right)^{*} \cong\left(\mathcal{F}_{\theta}\left(N^{\prime}\right) \otimes \mathcal{F}_{\theta}\left(N^{\prime}\right)\right)^{*} .
$$

If $T \in \mathcal{L}\left(\mathcal{F}_{\theta}\left(N^{\prime}\right), \mathcal{F}_{\theta}\left(N^{\prime}\right)^{*}\right)$ and $T^{K} \in\left(\mathcal{F}_{\theta}\left(N^{\prime}\right) \otimes \mathcal{F}_{\theta}\left(N^{\prime}\right)\right)^{*}$ are related under this isomorphism, we have

$$
\langle\langle T \varphi, \psi\rangle\rangle=\left\langle\left\langle T^{K}, \varphi \otimes \psi\right\rangle\right\rangle
$$

We call $T^{K}$ the kernel of $T$.

The symbol of $T \in \mathcal{L}\left(\mathcal{F}_{\theta}\left(N^{\prime}\right), \mathcal{F}_{\theta}\left(N^{\prime}\right)^{*}\right)$ is by definition a $\mathbb{C}$-valued function on $N \times N$ defined by

$$
\sigma(T)(\xi, \eta)=\left\langle\left\langle T e_{\xi}, e_{\eta}\right\rangle\right\rangle e^{-\langle\xi, \eta\rangle}, \quad \xi, \eta \in N .
$$

Then, every operator in $\mathcal{L}\left(\mathcal{F}_{\theta}\left(N^{\prime}\right), \mathcal{F}_{\theta}\left(N^{\prime}\right)^{*}\right)$ is uniquely determined by its symbol since the exponential vectors $e_{\xi}$ span a dense subspace of $\mathcal{F}_{\theta}\left(N^{\prime}\right)$. Therefore, in a similar manner to the characterization theorem 2.2 for distributions in $\mathcal{F}_{\theta}\left(N^{\prime}\right)^{*}$, we have the following characterization theorem for operators.

THEOREM 2.3. The symbol map yields a topological isomorphism between

$$
\mathcal{L}\left(\mathcal{F}_{\theta}\left(N^{\prime}\right), \mathcal{F}_{\theta}\left(N^{\prime}\right)^{*}\right) \quad \text { and } \quad \mathcal{G}_{\theta^{*}}(N \oplus N) .
$$

More precisely, we have the following isomorphisms

$$
\mathcal{L}\left(\mathcal{F}_{\theta}\left(N^{\prime}\right), \mathcal{F}_{\theta}\left(N^{\prime}\right)^{*}\right) \rightarrow \mathcal{G}_{\theta^{*}}(N \oplus N) \rightarrow G_{\theta}\left(N^{\prime}\right) \otimes G_{\theta}\left(N^{\prime}\right) \equiv G_{\theta}\left((N \oplus N)^{\prime}\right)
$$




$$
T \mapsto \sigma(T)(\xi, \eta)=\sum_{l, m}\left\langle\kappa_{l, m}, \eta^{\otimes l} \otimes \xi^{\otimes m}\right\rangle \mapsto K=\left(\kappa_{l, m}\right)_{l, m}
$$

REMARK 2.4. It is noteworthy that the symbol transform defined by (2.8) is sometimes called the Wick symbol [8]. This is more like the standard notion of a symbol of a pseudo-differential operator (see e.g. [20]). In fact, we found that our definition is more convenient for the convolution product $\sigma\left(T_{1} * T_{2}\right)=\sigma\left(T_{1}\right) \sigma\left(T_{2}\right)$ and for the connection between the Lévy Laplacian acting on operators and the quadratic quantum white noise, (see Section 5). This is due to the peculiar feature of the so-called the quantum Lévy-type Laplacian: it receives a rigorous meaning of second order differential operator with respect to a suitable notion of derivations on some Lie algebra of quantum white noise vector fields. A further detailed study in this direction will appear in a future paper.

Let $y \in N^{\prime}$ and $\varphi \in \mathcal{F}_{\theta}\left(N^{\prime}\right)$. We define the holomorphic derivative of $\varphi(z)=$ $\sum_{n=0}^{\infty}\left\langle z^{\otimes n}, \varphi_{n}\right\rangle$ at a point $z \in N^{\prime}$ by

$$
D_{y} \varphi(z)=\sum_{n=0}^{\infty}(n+1)\left\langle z^{\otimes n}, y \otimes_{1} \varphi_{n+1}\right\rangle,
$$

where $\otimes_{1}$ is the right contraction of tensor product of degree 1 (see [21]). It is known that $D_{y} \in \mathcal{L}\left(\mathcal{F}_{\theta}\left(N^{\prime}\right), \mathcal{F}_{\theta}\left(N^{\prime}\right)\right)$ and hence $D_{y}^{*} \in \mathcal{L}\left(\mathcal{F}_{\theta}\left(N^{\prime}\right)^{*}, \mathcal{F}_{\theta}\left(N^{\prime}\right)^{*}\right)$. If, in particular, $E=\mathcal{S}(\mathbb{R}), H=L^{2}(\mathbb{R}, d t)$ and $E^{\prime}=\mathcal{S}^{\prime}(\mathbb{R})$ (the Schwartz distributions space), we may define

$$
a_{t}=D_{\delta_{t}}, \quad t \in \mathbb{R}
$$

and denote its adjoint operator by $a_{t}^{*}$. Then the operators $a_{t} \in \mathcal{L}\left(\mathcal{F}_{\theta}\left(N^{\prime}\right), \mathcal{F}_{\theta}\left(N^{\prime}\right)\right)$ and $a_{t}^{*} \in \mathcal{L}\left(\mathcal{F}_{\theta}\left(N^{\prime}\right)^{*}, \mathcal{F}_{\theta}\left(N^{\prime}\right)^{*}\right)$ are, respectively, called the annihilation operator and the creation operator at a point $t \in \mathbb{R}$. In all the remainder of this paper we shall consider the above Schwartz standard Gelfand triple when needed.

It is shown (see [16], [23]) that $\mathcal{G}_{\theta^{*}}(N \oplus N)$ is closed under pointwise multiplication, then the convolution product $T=T_{1} * T_{2}$ of $T_{1}, T_{2} \in \mathcal{L}\left(\mathcal{F}_{\theta}\left(N^{\prime}\right), \mathcal{F}_{\theta}\left(N^{\prime}\right)^{*}\right)$ is defined as the unique $T \in \mathcal{L}\left(\mathcal{F}_{\theta}\left(N^{\prime}\right), \mathcal{F}_{\theta}\left(N^{\prime}\right)^{*}\right)$ such that

$$
\sigma(T)=\sigma\left(T_{1} * T_{2}\right):=\sigma\left(T_{1}\right) \sigma\left(T_{2}\right) .
$$

We next recall the following useful result.

THEOREM 2.5 ([6]). Let $\varphi$ be the Young function given by

$$
\varphi(x)=\left(e^{\theta^{*}}-1\right)^{*}(x), \quad x \in \mathbb{R}_{+} .
$$

Then, for any $T \in \mathcal{L}\left(\mathcal{F}_{\theta}\left(N^{\prime}\right), \mathcal{F}_{\theta}\left(N^{\prime}\right)^{*}\right)$, the convolution exponential

$$
\exp ^{*} T:=\sum_{n=0}^{\infty} \frac{1}{n !} T^{* n}
$$

converges in $\mathcal{L}\left(\mathcal{F}_{\varphi}\left(N^{\prime}\right), \mathcal{F}_{\varphi}\left(N^{\prime}\right)^{*}\right)$.

3. A quantum Lévy-type Laplacian. In this section, based on the paper [1], we shall review the so-called quantum Lévy-type Laplacian. 
Let $E_{1}, E_{2}$ be two real nuclear Fréchet spaces with complexifications $N_{1}$ and $N_{2}$ as before. A function $F: E_{1} \oplus E_{2} \rightarrow \mathbb{R}$ is called of class $C^{2}\left(E_{1} \oplus E_{2}\right)$ if there exist two continuous maps $\xi \oplus \eta \mapsto F^{\prime}(\xi, \eta) \in\left(E_{1} \oplus E_{2}\right)^{\prime}$ and

$$
\xi \oplus \eta \mapsto F^{\prime \prime}(\xi, \eta) \in \mathcal{L}\left(\left(E_{1} \oplus E_{2}\right),\left(E_{1} \oplus E_{2}\right)^{\prime}\right) \cong\left(\left(E_{1} \oplus E_{2}\right)^{\otimes 2}\right)^{\prime}, \quad \xi \oplus \eta \in E_{1} \oplus E_{2},
$$

such that, for any $\xi \oplus \eta \in E_{1} \oplus E_{2}$, we have

$$
F((\xi, \eta)+(a, b))=F(\xi, \eta)+\left\langle F^{\prime}(\xi, \eta), a \oplus b\right\rangle+\frac{1}{2}\left\langle F^{\prime \prime}(\xi, \eta),(a \oplus b)^{\otimes 2}\right\rangle+\varepsilon(\xi, \eta),
$$

where the error term satisfies

$$
\lim _{\gamma \rightarrow 0} \frac{\varepsilon(\gamma(\xi, \eta))}{\gamma^{2}}=0, \quad \xi \oplus \eta \in E_{1} \oplus E_{2}
$$

and the nuclear kernel theorem is used into account. A $\mathbb{C}$-valued function $F: E_{1} \oplus E_{2} \rightarrow \mathbb{C}$ lies in $C^{2}\left(E_{1} \oplus E_{2}\right)$ if so do its real and imaginary parts. In that case, $F^{\prime}(\xi, \eta) \in\left(N_{1} \oplus N_{2}\right)^{\prime}$ and $F^{\prime \prime}(\xi, \eta) \in\left(\left(N_{1} \oplus N_{2}\right)^{\otimes 2}\right)^{\prime}$.

Fix an arbitrary sequence $\left\{e_{n} \oplus f_{m}\right\}_{n, m \in \mathbb{N}}$ in $E_{1} \oplus E_{2}$, i.e. two sequences $e=\left(e_{n}\right)_{n \in \mathbb{N}}$ and $f=\left(f_{m}\right)_{m \in \mathbb{N}}$ in $E_{1}$ and $E_{2}$, respectively. From the paper [1] we introduce the following definition.

Definition 3.1. Let $F: E_{1} \oplus E_{2} \rightarrow \mathbb{C}$ be an element of $C^{2}\left(E_{1} \oplus E_{2}\right)$. By a Laplacian of Lévy-type associated with the sequence $(e, f)=\left(e_{n} \oplus f_{m}\right)_{n, m \in \mathbb{N}}$ we mean the elliptic operator $\tilde{\triangle}_{L}^{Q}$ defined by

$$
\left(\tilde{\triangle}_{L}^{Q} F\right)(\xi, \eta):=\lim _{N_{1}, N_{2} \rightarrow \infty} \frac{1}{N_{1} N_{2}} \sum_{n=1}^{N_{1}} \sum_{m=1}^{N_{2}}\left\langle F^{\prime \prime}(\xi, \eta),\left(e_{n} \oplus f_{m}\right)^{\otimes 2}\right\rangle, \quad \xi \oplus \eta \in E_{1} \oplus E_{2},
$$

whenever the limit exists.

The above definition is a natural extension of the usual Lévy Laplacian $\tilde{\triangle}_{L}$ defined, with respect to the infinite sequence $e=\left\{e_{n}\right\}_{n \in \mathbb{N}} \subset E_{1}$, on a function $f \in C^{2}\left(E_{1}\right)$ by

$$
\left(\tilde{\Delta}_{L} f\right)(\xi):=\lim _{n \rightarrow \infty} \frac{1}{n} \sum_{j=1}^{n}\left\langle f^{\prime \prime}(\xi) e_{j}, e_{j}\right\rangle, \quad \xi \in E_{1},
$$

when the limit exists. To be more precise, if for example $E_{2}=\{0\}$ then $f_{m}=0$ for any $m \in \mathbb{N}$ and one can consider the identifications

$$
E_{1}=E_{1} \oplus\{0\}, \quad \xi=\xi \oplus 0 \text { and } F(\xi, 0)=F(\xi), \quad \forall \xi \in E_{1},
$$

then

$$
F^{\prime}(\xi, 0)=F^{\prime}(\xi) \text { and } F^{\prime \prime}(\xi, 0)=F^{\prime \prime}(\xi) .
$$

So, we get the following Lemma:

LEMMA 3.2. The restriction of the Laplacian of Lévy-type $\widetilde{\triangle}_{L}^{Q}$ associated with the sequence $(e, f)=\left(e_{n} \oplus f_{m}\right)_{n, m \in \mathbb{N}}$ to the subspace $E_{1} \equiv E_{1} \oplus\{0\} \quad$ (resp. $\left.E_{2} \equiv\{0\} \oplus E_{2}\right)$ gives rise to the usual Lévy Laplacian $\tilde{\triangle}_{L}$ on $E_{1}$ (resp. $E_{2}$ ) associated with the sequence $e=\left(e_{n}\right)_{n \in \mathbb{N}}$ (resp. $\left.f=\left(f_{m}\right)_{m \in \mathbb{N}}\right)$.

The above Lemma is crucial for the operator $\widetilde{\triangle}_{L}^{Q}$ acting on functions in two variables. 
Recall that for $T \in \mathcal{L}\left(\mathcal{F}_{\theta}\left(N^{\prime}\right), \mathcal{F}_{\theta}\left(N^{\prime}\right)^{*}\right)$, the symbol transform $\sigma(T)$ belongs to $\mathcal{G}_{\theta^{*}}(N \oplus N)$. In particular, $\sigma(T) \in C^{2}(N \oplus N)$. Let $e=\left(e_{n}\right)_{n \in \mathbb{N}}$ and $f=\left(f_{m}\right)_{m \in \mathbb{N}}$ be two arbitrary sequences in $N$ and let $\mathcal{D}_{L}^{Q}(N)$ denote the space of all $T \in \mathcal{L}\left(\mathcal{F}_{\theta}\left(N^{\prime}\right), \mathcal{F}_{\theta}\left(N^{\prime}\right)^{*}\right)$ such that $\sigma(T) \in \operatorname{Dom}\left(\tilde{\triangle}_{L}^{Q}\right)$ and $\tilde{\triangle}_{L}^{Q}(\sigma(T)) \in \mathcal{G}_{\theta^{*}}(N \oplus N)$.

Definition 3.3. The quantum Lévy-type Laplacian $\triangle_{L}^{Q}$, acting on $\mathcal{D}_{L}^{Q}(N)$, is defined by

$$
\triangle_{L}^{Q}(T)=\sigma^{-1}\left(\tilde{\triangle}_{L}^{Q}(\sigma(T))\right), \quad T \in \mathcal{D}_{L}^{Q}(N)
$$

4. Basic examples. In the remainder of this Section we fix two sequences $e=\left(e_{n}\right)_{n \in \mathbb{N}}$ and $f=\left(f_{m}\right)_{m \in \mathbb{N}}$ of vectors in $E$. We denote by $N_{L, e}^{\prime}$ and $N_{L, f}^{\prime}$ the sets of all vectors $z \in N^{\prime}$ and $\omega \in N^{\prime}$ such that

$$
\begin{aligned}
\|z\|_{L, e}^{2} & :=\lim _{N_{1} \rightarrow \infty} \frac{1}{N_{1}} \sum_{n=1}^{N_{1}}\left\langle z, e_{n}\right\rangle^{2}<\infty, \\
\|\omega\|_{L, f}^{2} & :=\lim _{N_{2} \rightarrow \infty} \frac{1}{N_{2}} \sum_{m=1}^{N_{2}}\left\langle\omega, f_{m}\right\rangle^{2}<\infty,
\end{aligned}
$$

respectively.

4.1. Translation operators. Let $y \in N^{\prime}$. For $\varphi \in \mathcal{F}_{\theta}\left(N^{\prime}\right)$, the translation operator $\tau_{y} \varphi$ of $\varphi$ by $y$ is defined by

$$
\tau_{y} \varphi(x)=\varphi(x+y), \quad x \in N^{\prime} .
$$

It is easy to check that $\tau_{y}$ is a continuous linear operator from $\mathcal{F}_{\theta}\left(N^{\prime}\right)$ into itself. We fix $y \in N^{\prime}$. Then, for $\xi \in N$, we see that $\tau_{y} e_{\xi}=e^{\langle y, \xi\rangle} e_{\xi}$. Hence, by definition, one has

$$
\sigma\left(\tau_{y}\right)(\xi, \eta)=\left\langle\left\langle\tau_{y} e_{\xi}, e_{\eta}\right\rangle\right\rangle e^{-\langle\xi, \eta\rangle}=e^{\langle y, \xi\rangle}, \quad \xi, \eta \in N
$$

Therefore, for any $n, m \in \mathbb{N}$

$$
\left\langle\sigma\left(\tau_{y}\right)^{\prime \prime}(\xi, \eta),\left(e_{n} \oplus f_{m}\right)^{\otimes 2}\right\rangle=\left\langle y, e_{n}\right\rangle^{2} \sigma\left(\tau_{y}\right)(\xi, \eta), \quad \xi, \eta \in N .
$$

Proposition 4.1. Let $y \in N^{\prime}$. Then $\tau_{y} \in \mathcal{D}_{L}^{Q}(N)$ if and only if $y \in N_{L, e}^{\prime}$ and, in this case, $\tau_{y}$ is an eigenvector for $\triangle_{L}^{Q}$ in such a way that

$$
\triangle_{L}^{Q}\left(\tau_{y}\right)=\|y\|_{L, e}^{2} \tau_{y}
$$

Proof. The proof follows from (4.3) and the definition of $\triangle_{L}^{Q}$.

4.2. Integral kernel operators. Let a pair $l, m \in \mathbb{N}$ and a distribution $\kappa_{l, m} \in\left(N^{\otimes(l+m)}\right)^{\prime}$ be given. The function $\Theta_{l, m}:(\xi, \eta) \mapsto\left\langle\kappa_{l, m}, \eta^{\otimes l} \otimes \xi^{\otimes m}\right\rangle$ is of polynomial growth, then it belongs to $\mathcal{G}_{\theta^{*}}(N \oplus N)$. Then we shall denote by $T_{l, m}\left(\kappa_{l, m}\right) \in \mathcal{L}\left(\mathcal{F}_{\theta}\left(N^{\prime}\right), \mathcal{F}_{\theta}\left(N^{\prime}\right)^{*}\right)$ the unique operator associated to $\Theta_{l, m}$ via the symbol transform. We employ the formal integral expression:

$$
T_{l, m}\left(\kappa_{l, m}\right)=\int_{\mathbb{R}^{l+m}} \kappa_{l, m}\left(s_{1}, \ldots, s_{l}, t_{1}, \ldots, t_{m}\right) a_{s_{1}}^{*} \ldots a_{s_{l}}^{*} a_{t_{1}} \ldots a_{t_{m}} d s_{1} \ldots d s_{l} d t_{1} \ldots d t_{m}
$$

and call it the integral kernel operator with kernel $\kappa_{l, m}$ (see e.g. [21]). By definition, the symbol of $T_{l, m}\left(\kappa_{l, m}\right)$ is given by

$$
\sigma\left(T_{l, m}\left(\kappa_{l, m}\right)\right)(\xi, \eta)=\left\langle\kappa_{l, m}, \eta^{\otimes l} \otimes \xi^{\otimes m}\right\rangle, \quad \xi, \eta \in N .
$$


For simplicity, we put $F(\xi, \eta):=\left\langle\kappa_{l, m}, \eta^{\otimes l} \otimes \xi^{\otimes m}\right\rangle$. Then, by direct computation, for any $p, q \in \mathbb{N}$, we have

$$
\begin{aligned}
\left\langle F^{\prime \prime}(\xi, \eta),\left(e_{p} \oplus f_{q}\right)^{\otimes 2}\right\rangle= & l(l-1)\left\langle\kappa_{l, m},\left(\eta^{\otimes(l-2)} \hat{\otimes} f_{q}^{\otimes 2}\right) \otimes \xi^{\otimes m}\right\rangle \\
& +m(m-1)\left\langle\kappa_{l, m}, \eta^{\otimes l} \otimes\left(\xi^{\otimes(m-2)} \hat{\otimes} e_{p}^{\otimes 2}\right)\right\rangle \\
& +2 \operatorname{lm}\left\langle\kappa_{l, m},\left(\eta^{\otimes(l-1)} \hat{\otimes} f_{q}\right) \otimes\left(\xi^{\otimes(m-1)} \hat{\otimes} e_{p}\right)\right\rangle .
\end{aligned}
$$

This suggests the following:

TheOREm 4.2. Let $l, m \in \mathbb{N}$. Suppose that

$$
\kappa_{l, m}=z^{\otimes l} \otimes \omega^{\otimes m} \in\left(E^{\otimes l}\right)^{\prime} \otimes\left(E^{\otimes m}\right)^{\prime}
$$

and denote

$$
\kappa_{l-i, m-j}:=z^{\otimes(l-i)} \otimes \omega^{\otimes(m-j)}, \quad 0 \leq i \leq l, 0 \leq j \leq m .
$$

Then, $T_{l, m}\left(z^{\otimes l} \otimes \omega^{\otimes m}\right) \in \mathcal{D}_{L}^{Q}(N)$ if and only if $z \in E_{L, f}^{\prime}$ and $\omega \in E_{L, e}^{\prime}$ and in that case

$$
\begin{aligned}
& \triangle_{L}^{Q}\left(T_{l, m}\left(\kappa_{l, m}\right)\right)=l(l-1) T_{l-2, m}\left(\kappa_{l-2, m}\right)\|z\|_{L, f}^{2}+m(m-1) T_{l, m-2}\left(\kappa_{l, m-2}\right)\|\omega\|_{L, e}^{2} \\
& \quad+2 l m T_{l-1, m-1}\left(\kappa_{l-1, m-1}\right)\left\{\lim _{N_{1} \rightarrow \infty} \frac{1}{N_{1}} \sum_{p=1}^{N_{1}}\left\langle\omega, e_{p}\right\rangle\right\}\left\{\lim _{N_{2} \rightarrow \infty} \frac{1}{N_{2}} \sum_{q=1}^{N_{2}}\left\langle z, f_{q}\right\rangle\right\} .
\end{aligned}
$$

Proof. With our assumption (4.5), (4.4) becomes

$$
\begin{aligned}
\left\langle F^{\prime \prime}(\xi, \eta),\left(e_{p} \oplus f_{q}\right)^{\otimes 2}\right\rangle= & l(l-1)\left\langle\kappa_{l-2, m}, \eta^{\otimes(l-2)} \otimes \xi^{\otimes m}\right\rangle\left\langle z, f_{q}\right\rangle^{2} \\
& +m(m-1)\left\langle\kappa_{l, m-2}, \eta^{\otimes l} \otimes \xi^{\otimes(m-2)}\right\rangle\left\langle\omega, e_{p}\right\rangle^{2} \\
& +2 l m\left\langle\kappa_{l-1, m-1}, \eta^{\otimes(l-1)} \otimes \xi^{\otimes(m-1)}\right\rangle\left\langle z, f_{q}\right\rangle\left\langle\omega, e_{p}\right\rangle \\
= & l(l-1) \sigma\left(T_{l-2, m}\left(\kappa_{l-2, m}\right)\right)(\xi, \eta)\left\langle z, f_{q}\right\rangle^{2} \\
& +m(m-1) \sigma\left(T_{l, m-2}\left(\kappa_{l, m-2}\right)\right)(\xi, \eta)\left\langle\omega, e_{p}\right\rangle^{2} \\
& +2 l m \sigma\left(T_{l-1, m-1}\left(\kappa_{l-1, m-1}\right)\right)(\xi, \eta)\left\langle z, f_{q}\right\rangle\left\langle\omega, e_{p}\right\rangle .
\end{aligned}
$$

It follows that

$$
\begin{aligned}
& \triangle_{L}^{Q}\left(T_{l, m}\left(\kappa_{l, m}\right)\right) \\
& =l(l-1) T_{l-2, m}\left(\kappa_{l-2, m}\right)\|z\|_{L, f}^{2}+m(m-1) T_{l, m-2}\left(\kappa_{l, m-2}\right)\|\omega\|_{L, e}^{2} \\
& +2 l m T_{l-1, m-1}\left(\kappa_{l-1, m-1}\right)\left\{\lim _{N_{1}, N_{2} \rightarrow \infty} \frac{1}{N_{1} N_{2}} \sum_{p=1}^{N_{1}} \sum_{q=1}^{N_{2}}\left\langle\omega, e_{p}\right\rangle\left\langle z, f_{q}\right\rangle\right\},
\end{aligned}
$$

when the right side of (4.7) makes sense. Now if $\|z\|_{L, f}^{2}<\infty$ and $\|\omega\|_{L, e}^{2}<\infty$, by using the Schwarz inequality, we have

$$
\lim _{N_{1}, N_{2} \rightarrow \infty} \frac{1}{N_{1} N_{2}} \sum_{p=1}^{N_{1}} \sum_{q=1}^{N_{2}}\left\langle\omega, e_{p}\right\rangle\left\langle z, f_{q}\right\rangle<\infty
$$

and the statement follows.

REMARK 4.3. Suppose that $E=\mathcal{S}(\mathbb{R}), H=L^{2}(\mathbb{R}, d t)$ and $E^{\prime}=\mathcal{S}^{\prime}(\mathbb{R})$ (the Schwartz distribution space). In the notations of the above Theorem, by taking $z=\omega=\delta_{t}$, one can 
found the action of $\triangle_{L}^{Q}$ on higher powers of quantum white noises. To be more precise let $l, m \in \mathbb{N}$ and $t \in \mathbb{R}_{+}$. For $T=a_{t}^{*} a_{t}^{m} \in \mathcal{L}\left(\mathcal{F}_{\theta}\left(N^{\prime}\right), \mathcal{F}_{\theta}\left(N^{\prime}\right)^{*}\right)$, one has

$$
\begin{aligned}
\triangle_{L}^{Q}\left(a_{t}^{*^{l}} a_{t}^{m}\right)= & m(m-1) a_{t}^{*^{l}} a_{t}^{m-2}\left\|\delta_{t}\right\|_{L, e}^{2}+l(l-1) a_{t}^{*^{l-2}} a_{t}^{m}\left\|\delta_{t}\right\|_{L, f}^{2} \\
& +2 \operatorname{lm} a_{t}^{*^{l-1}} a_{t}^{m-1}\left(\lim _{N_{1}, N_{2} \rightarrow \infty} \frac{1}{N_{1} N_{2}} \sum_{p=1}^{N_{1}} \sum_{q=1}^{N_{2}}\left\langle\delta_{t}, e_{p}\right\rangle\left\langle\delta_{t}, f_{q}\right\rangle\right)
\end{aligned}
$$

when the limits exist.

It is well-known (see [18]) that if $e=f=\left(e_{n}\right)_{n \in \mathbb{N}} \subset E$ is the complete orthonormal basis consisting of Hermite functions of $H$, then $\sup _{t \in \mathbb{R}}\left|\left\langle\delta_{t}, e_{n}\right\rangle\right|=O\left(n^{-1 / 12}\right)$. Therefore,

$$
\sum_{n=1}^{N_{1}}\left\langle\delta_{t}, e_{n}\right\rangle^{2} \approx \sum_{n=1}^{N_{1}} n^{-1 / 6}=O\left(N_{1}^{5 / 6}\right)
$$

and therefore

$$
\lim _{N_{1} \rightarrow \infty} \frac{1}{N_{1}} \sum_{n=1}^{N_{1}}\left\langle\delta_{t}, e_{n}\right\rangle^{2}=\left\|\delta_{t}\right\|_{L, e}^{2}=0 .
$$

In conclusion, the above relation (4.8) becomes

$$
\triangle_{L}^{Q}\left(a_{t}^{*^{l}} a_{t}^{m}\right)=0, \quad \forall l, m \in \mathbb{N} .
$$

So, all the higher powers of quantum white noises are eigenvectors of the quantum Lévytype Laplacian $\triangle_{L}^{Q}$ for the eigenvalue 0 .

4.3. Gross Laplacian. The Gross Laplacian (see [15], [18]) is the operator defined by its Fock expansion as follows:

$$
\triangle_{G}=T_{0,2}(\tau)=\int \tau(s, t) a_{s} a_{t} d s d t
$$

where $\tau \in\left(N^{\otimes 2}\right)^{\prime}$ is the trace defined by

$$
\langle\tau, \xi \otimes \eta\rangle=\langle\xi, \eta\rangle, \quad \xi, \eta \in N .
$$

It is well-known that $\triangle_{G}$ is a linear operator from $\mathcal{F}_{\theta}\left(N^{\prime}\right)$ into itself. We describe the action of the quantum Lévy-type Laplacian $\triangle_{L}^{Q}$ on this infinite dimensional classical Laplacian.

Proposition 4.4. The quantum Lévy-type Laplacian $\triangle_{L}^{Q}$ acts on the Gross Laplacian $\triangle_{G}$ as follows:

$$
\triangle_{L}^{Q}\left(\triangle_{G}\right)=2 \lim _{N_{1} \rightarrow \infty} \frac{1}{N_{1}} \sum_{n=1}^{N_{1}}\left\langle e_{n}, e_{n}\right\rangle I
$$

if the limit exists. In particular, if $e=\left(e_{n}\right)_{n \in \mathbb{N}}$ is an orthonormal system, then $\triangle_{G} \in$ $\mathcal{D}_{L}^{Q}(N)$ and we have

$$
\triangle_{L}^{Q}\left(\triangle_{G}\right)=2 I
$$

where $I$ is the identity operator of $\mathcal{L}\left(\mathcal{F}_{\theta}\left(N^{\prime}\right), \mathcal{F}_{\theta}\left(N^{\prime}\right)\right)$.

Proof. By direct computation we obtain

$$
\sigma\left(\triangle_{G}\right)(\xi, \eta)=\left\langle\tau, \xi^{\otimes 2}\right\rangle=\langle\xi, \xi\rangle, \quad \xi, \eta \in N .
$$


Then, for any $n, m \in \mathbb{N}$

$$
\left\langle\sigma\left(\triangle_{G}\right)^{\prime \prime}(\xi, \eta),\left(e_{n} \oplus f_{m}\right)^{\otimes 2}\right\rangle=2\left\langle e_{n}, e_{n}\right\rangle, \quad \xi, \eta \in N .
$$

Therefore

$$
\tilde{\triangle}_{L}^{Q}\left(\sigma\left(\triangle_{G}\right)\right)(\xi, \eta)=2 \lim _{N_{1} \rightarrow \infty} \frac{1}{N_{1}} \sum_{n=1}^{N_{1}}\left\langle e_{n}, e_{n}\right\rangle
$$

if the limit exists. To conclude, it is sufficient to remark that $\sigma(I)=1$.

4.4. Convolution operators. By a convolution operator on the test function space $\mathcal{F}_{\theta}\left(N^{\prime}\right)$ we mean a continuous linear operator from $\mathcal{F}_{\theta}\left(N^{\prime}\right)$ into itself which commutes with all translation operators $\tau_{x}, x \in N^{\prime}$.

We define the convolution $\Phi * \varphi$ of a distribution $\Phi \in \mathcal{F}_{\theta}\left(N^{\prime}\right)^{*}$ and a test function $\varphi \in \mathcal{F}_{\theta}\left(N^{\prime}\right)$ to be the function

$$
(\Phi * \varphi)(x)=\left\langle\left\langle\Phi, \tau_{x} \varphi\right\rangle\right\rangle, \quad x \in N^{\prime} .
$$

Note, in particular, that the distribution $\Phi \in \mathcal{F}_{\theta}\left(N^{\prime}\right)^{*}$ acts on a test exponential function by:

$$
\Phi * e_{\xi}=(\mathcal{L} \Phi)(\xi) e_{\xi}, \quad \xi \in N .
$$

Direct calculation shows that $\Phi * \varphi \in \mathcal{F}_{\theta}\left(N^{\prime}\right)$, for any $\varphi \in \mathcal{F}_{\theta}\left(N^{\prime}\right)$, and that the mapping $T_{\Phi}$ defined by

$$
T_{\Phi}: \varphi \mapsto \Phi * \varphi, \quad \varphi \in \mathcal{F}_{\theta}\left(N^{\prime}\right)
$$

is a convolution operator on $\mathcal{F}_{\theta}\left(N^{\prime}\right)$. Conversely, it was proved in [6] that all convolution operators on $\mathcal{F}_{\theta}\left(N^{\prime}\right)$ occur this way, i.e., if $T$ is a convolution operator on $\mathcal{F}_{\theta}\left(N^{\prime}\right)$, then there exists a unique $\Phi \in \mathcal{F}_{\theta}\left(N^{\prime}\right)^{*}$ such that $T=T_{\Phi}$, or equivalently,

$$
T(\varphi)=T_{\Phi}(\varphi)=\Phi * \varphi, \quad \varphi \in \mathcal{F}_{\theta}\left(N^{\prime}\right) .
$$

Suppose $\Phi_{1}, \Phi_{2} \in \mathcal{F}_{\theta}\left(N^{\prime}\right)^{*}$. Let $T_{\Phi_{1}}$ and $T_{\Phi_{2}}$ be the convolution operators given by $\Phi_{1}$ and $\Phi_{2}$, respectively, as in Equation (4.10). It is clear that the composition $T_{\Phi_{1}} \circ T_{\Phi_{2}}$ is also a convolution operator on $\mathcal{F}_{\theta}\left(N^{\prime}\right)$. Hence there exists a unique distribution, denoted by $\Phi_{1} * \Phi_{2}$, in $\mathcal{F}_{\theta}\left(N^{\prime}\right)^{*}$ such that

$$
T_{\Phi_{1}} \circ T_{\Phi_{2}}=T_{\Phi_{1} * \Phi_{2}}
$$

The distribution $\Phi_{1} * \Phi_{2}$ in Equation (4.11) is called the convolution of $\Phi_{1}$ and $\Phi_{2}$. From Proposition 1 of the paper [6] we have the following equality for any $\Phi_{1}, \Phi_{2} \in \mathcal{F}_{\theta}\left(N^{\prime}\right)^{*}$ :

$$
\mathcal{L}\left(\Phi_{1} * \Phi_{2}\right)=\mathcal{L} \Phi_{1} \mathcal{L} \Phi_{2} .
$$

This proves, in particular, that the space $\mathcal{L}_{c}$ of all convolution operators on $\mathcal{F}_{\theta}\left(N^{\prime}\right)$ is a commutative subalgebra of $\mathcal{L}\left(\mathcal{F}_{\theta}\left(N^{\prime}\right), \mathcal{F}_{\theta}\left(N^{\prime}\right)\right)$.

In the white noise theory the classical Lévy Laplacian is defined through the Laplace transform. For $\Phi \in \mathcal{F}_{\theta}\left(N^{\prime}\right)^{*}$, since $\mathcal{L} \Phi \in \mathcal{G}_{\theta^{*}}(N)$, we have $\mathcal{L} \Phi \in C^{2}(N)$. Let $\mathcal{D}_{L}(N)$ denote the space of all $\Phi \in \mathcal{F}_{\theta}\left(N^{\prime}\right)^{*}$ such that

$$
\tilde{\triangle}_{L}(\mathcal{L} \Phi)(\xi):=\lim _{n \rightarrow \infty} \frac{1}{n} \sum_{j=1}^{n}\left\langle(\mathcal{L} \Phi)^{\prime \prime}(\xi), e_{j} \otimes e_{j}\right\rangle
$$


exists for any $\xi \in N$, and $\tilde{\triangle}_{L}(\mathcal{L}(\Phi)) \in \mathcal{G}_{\theta^{*}}(N)$ (where $e=\left(e_{n}\right)_{n \in \mathbb{N}}$ is an arbitrary infinite sequence of elements of $N)$. Then the Lévy Laplacian acts on $\Phi \in \mathcal{D}_{L}(N)$ by

$$
\triangle_{L} \Phi:=\mathcal{L}^{-1}\left(\tilde{\triangle}_{L}(\mathcal{L} \Phi)\right) \in \mathcal{F}_{\theta}\left(N^{\prime}\right)^{*} .
$$

The next Proposition gives a connection between the classical Lévy Laplacian $\tilde{\triangle}_{L}$ and the quantum Lévy-type Laplacian $\widetilde{\triangle}_{L}^{Q}$ on the algebra of convolution operators.

Proposition 4.5. For any $\Phi \in \mathcal{D}_{L}(N)$ we have $T_{\Phi} \in \mathcal{D}_{L}^{Q}(N)$ and

$$
\tilde{\triangle}_{L}^{Q}\left(\sigma\left(T_{\Phi}\right)\right)(\xi, \eta)=\tilde{\triangle}_{L}(\mathcal{L} \Phi)(\xi), \quad \xi, \eta \in N
$$

Proof. Let $\Phi \in \mathcal{D}_{L}(N)$. By definition and the relation (4.9) we have, for any $\xi, \eta \in N$,

$$
\sigma\left(T_{\Phi}\right)(\xi, \eta)=\left\langle\left\langle T_{\Phi} e_{\xi}, e_{\eta}\right\rangle\right\rangle e^{-\langle\xi, \eta\rangle}=\left\langle\left\langle\Phi * e_{\xi}, e_{\eta}\right\rangle\right\rangle e^{-\langle\xi, \eta\rangle}=(\mathcal{L} \Phi)(\xi) .
$$

Then, using the definitions of $\tilde{\triangle}_{L}^{Q}$ and $\tilde{\triangle}_{L}$, one has

$$
\begin{aligned}
\tilde{\triangle}_{L}^{Q}\left(\sigma\left(T_{\Phi}\right)\right)(\xi, \eta) & =\lim _{N_{1}, N_{2} \rightarrow \infty} \frac{1}{N_{1} N_{2}} \sum_{n=1}^{N_{1}} \sum_{m=1}^{N_{2}}\left\langle\sigma\left(T_{\Phi}\right)^{\prime \prime}(\xi, \eta),\left(e_{n} \oplus f_{m}\right)^{\otimes 2}\right\rangle \\
& =\lim _{N_{1}, N_{2} \rightarrow \infty} \frac{1}{N_{1} N_{2}} \sum_{n=1}^{N_{1}} \sum_{m=1}^{N_{2}}\left\langle(\mathcal{L} \Phi)^{\prime \prime}(\xi),\left(e_{n} \oplus f_{m}\right)^{\otimes 2}\right\rangle \\
& =\lim _{N_{1}, N_{2} \rightarrow \infty} \frac{1}{N_{1} N_{2}} \sum_{n=1}^{N_{1}} \sum_{m=1}^{N_{2}}\left\langle(\mathcal{L} \Phi)^{\prime \prime}(\xi), e_{n}^{\otimes 2}\right\rangle \\
& =\lim _{N_{1} \rightarrow \infty} \frac{1}{N_{1}} \sum_{n=1}^{N_{1}}\left\langle(\mathcal{L} \Phi)^{\prime \prime}(\xi), e_{n}^{\otimes 2}\right\rangle \\
& =\tilde{\triangle}_{L}(\mathcal{L} \Phi)(\xi) .
\end{aligned}
$$

This proves the statement.

5. Quadratic quantum white noise and the quantum Lévy-type Laplacian. In this Section we give, in the frame of white noise calculus, a good connection between square of quantum white noise and the quantum Lévy-type laplacian $\triangle_{L}^{Q}$, and we discuss the related heat equation.

In the remainder, we assume that $E=S(\mathbb{R}), H=L^{2}(\mathbb{R}, d t)$, and $E^{\prime}=S^{\prime}(\mathbb{R})$.

Following [9], any continuous map $t \mapsto T_{t} \in \mathbb{H}=L^{2}\left(E^{\prime}, \mu, \mathbb{C}\right)$ defined on an interval can be considered as a usual (classical) stochastic process. Hence, any continuous function whose domain is an interval of $\mathbb{R}$ and a range in $\mathcal{F}_{\theta}\left(N^{\prime}\right)^{*}$ can be called a (generalized) classic stochastic process. In a similar way, in the frame of white noise analysis, a (generalized) quantum stochastic process is a continuous mapping of a subset of $\mathbb{R}$ onto $\mathcal{L}\left(\mathcal{F}_{\theta}\left(N^{\prime}\right), \mathcal{F}_{\theta}\left(N^{\prime}\right)^{*}\right)$.

We now focus on the convolution differential equation with quadratic quantum white noise as coefficients:

$$
\frac{d T}{d t}=\left(a_{t}^{2}+a_{t}^{* 2}\right) * T, \quad T_{0}=I .
$$


Define a quantum stochastic process $\left\{Z_{t}\right\}$ by

$$
Z_{t}=\int_{0}^{t}\left(a_{s}^{2}+a_{s}^{* 2}\right) d s
$$

Since $\mathcal{L}\left(\mathcal{F}_{\theta}\left(N^{\prime}\right), \mathcal{F}_{\theta}\left(N^{\prime}\right)^{*}\right)$ is a commutative algebra with respect to the convolution product (see (2.9)), the formal solution to (5.1) is given by the convolution exponential:

$$
T_{t}=\exp ^{*}\left(Z_{t}\right)=\sum_{n=0}^{\infty} \frac{1}{n !}\left(Z_{t}\right)^{{ }_{n}} .
$$

The symbol of (5.2) is given by

$$
\sigma\left(T_{t}\right)(\xi, \eta)=\sum_{n=0}^{\infty} \frac{1}{n !}\left(\sigma\left(Z_{t}\right)\right)^{n}(\xi, \eta)=\exp \left\{\sigma\left(Z_{t}\right)(\xi, \eta)\right\}, \quad \xi, \eta \in N .
$$

But, by definition we have

$$
\sigma\left(Z_{t}\right)(\xi, \eta)=\int_{0}^{t}\left\langle\left\langle\left(a_{s}^{2}+a_{s}^{* 2}\right) e_{\xi}, e_{\eta}\right\rangle\right\rangle e^{-\langle\xi, \eta\rangle} d s=\int_{0}^{t}\left(\xi(s)^{2}+\eta(s)^{2}\right) d s .
$$

Hence, (5.3) becomes

$$
\sigma\left(T_{t}\right)(\xi, \eta)=\exp \left\{\int_{0}^{t}\left(\xi(s)^{2}+\eta(s)^{2}\right) d s\right\}, \quad \xi, \eta \in N .
$$

THEOREM 5.1. The quadratic quantum white noise convolution differential equation (5.1) has a unique solution $\left\{T_{t}\right\}$ in $\mathcal{L}\left(\mathcal{F}_{\theta}\left(N^{\prime}\right), \mathcal{F}_{\theta}\left(N^{\prime}\right)^{*}\right)$ given by (5.2) and the map $t \rightarrow T_{t}$ is continuous.

Proof. Let $p \geq 0$ and $m>0$. Then, using (5.4) we have the estimates

$$
\begin{aligned}
\left|\sigma\left(T_{t}\right)(\xi, \eta)\right| \exp \left(-\theta^{*}\left(m|\xi|_{p}\right)\right. & \left.-\theta^{*}\left(m|\eta|_{p}\right)\right) \\
& =\exp \left\{\int_{0}^{t}\left(\xi(s)^{2}+\eta(s)^{2}\right) d s-\theta^{*}\left(m|\xi|_{p}\right)-\theta^{*}\left(m|\eta|_{p}\right)\right\} \\
& \leq \exp \left\{|\xi|_{0}^{2}-\theta^{*}\left(m|\xi|_{p}\right)\right\} \times \exp \left\{|\eta|_{0}^{2}-\theta^{*}\left(m|\eta|_{p}\right)\right\} \\
& \leq \exp \left\{|\xi|_{p}^{2}-\theta^{*}\left(m|\xi|_{p}\right)\right\} \times \exp \left\{|\eta|_{p}^{2}-\theta^{*}\left(m|\eta|_{p}\right)\right\} .
\end{aligned}
$$

We need the following statement:

FACT. (1) For $0 \leq \delta \leq 1, x \geq 0$, we have

$$
\delta \theta^{*}(x) \geq \theta^{*}(\delta x)
$$

(2) For $\delta \geq 1, x \geq 0$, we have

$$
\delta \theta^{*}(x) \leq \theta^{*}(\delta x) .
$$

Indeed, $\theta^{*}$ is a Young function, so $\theta^{*}$ is convex, then for any $0 \leq \delta \leq 1, x \geq 0$

$$
\theta^{*}(\delta x-(1-\delta) 0) \leq \delta \theta^{*}(x)+(1-\delta) \theta^{*}(0)=\delta \theta^{*}(x) .
$$

Hence $\delta \theta^{*}(x) \geq \theta^{*}(\delta x)$. This proves (5.5). The relation (5.6) follows by changing $\delta$ to $\frac{1}{\delta}$. The condition (2.7) says that there exists $\delta>0$ small enough $(0<\delta \leq 1)$ such that 
$\frac{1}{\delta} \theta^{*}\left(|\xi|_{p}\right) \geq|\xi|_{p}^{2}$. Then, taking (5.6) into account, one has, for any $p \geq 0$ and $m=1 / \delta>0$ $\left\|\sigma\left(T_{t}\right)\right\|_{\theta^{*}, p, m}<\infty$.

This proves that $\sigma\left(T_{t}\right) \in \mathcal{G}_{\theta^{*}}(N \oplus N)$, or equivalently, $T_{t} \in \mathcal{L}\left(\mathcal{F}_{\theta}\left(N^{\prime}\right), \mathcal{F}_{\theta}\left(N^{\prime}\right)^{*}\right)$. The continuity of the map $t \rightarrow T_{t}$ is straightforward.

REMARK 5.2. From the proof of the above theorem we see that the range of the solution $\left\{T_{t}\right\}$ of $(5.1)$ is contained in an algebra which is singular to the original $\mathcal{L}(\mathbb{H}, \mathbb{H})$, so that $\left\{T_{t}\right\}$ is a generalized quantum stochastic process.

We fix two infinite sequences $e=\left(e_{n}\right)_{n \in \mathbb{N}}$ and $f=\left(f_{m}\right)_{m \in \mathbb{N}}$ in $N$ satisfying the following properties:

(j) for any $\xi, \eta, \in N$

$$
\lim _{N_{1}, N_{2} \rightarrow \infty} \frac{1}{N_{1} N_{2}} \sum_{n=1}^{N_{1}} \sum_{m=1}^{N_{2}}\left(\int_{0}^{\alpha} e_{n}(u) \xi(u) d u+\int_{0}^{\alpha} f_{m}(u) \eta(u) d u\right)^{2}=0 .
$$

$(\mathbf{j j})$

$$
\lim _{N_{1} \rightarrow \infty} \frac{1}{N_{1}} \sum_{n=1}^{N_{1}} \int_{0}^{\alpha} e_{n}^{2}(u) d u=\lim _{N_{2} \rightarrow \infty} \frac{1}{N_{2}} \sum_{m=1}^{N_{2}} \int_{0}^{\alpha} f_{m}^{2}(u) d u=\alpha .
$$

Here $\alpha$ is a fixed positive real number.

THEOREM 5.3. Let $\left\{T_{s}\right\}$ be the generalized quantum stochastic process solution of the quadratic quantum white noise convolution differential equation (5.1). Let $\alpha \geq 0$. Assume that the above conditions $(\mathbf{j})$ and $(\mathbf{j j})$ are satisfied. Then $T_{\alpha}$ is an eigenfunction of the Lévy-type Laplacian $\triangle_{L}^{Q}$ such that

$$
\triangle_{L}^{Q} T_{\alpha}=4 \alpha T_{\alpha}
$$

Moreover, for $\gamma \in \mathbb{C}, L_{t}=e^{2 \gamma t} T_{\alpha}$ satisfies the quantum white noise heat equation

$$
\frac{\partial L}{\partial t}=\frac{\gamma}{2} \triangle_{L}^{Q} L, \quad L(0)=T_{\alpha}
$$

Proof. From (5.4) we have, for each $\xi, \eta \in N$,

$$
\sigma(T)(\xi, \eta)=\exp \left\{\int_{0}^{t}\left(\xi(s)^{2}+\eta(s)^{2}\right) d s\right\} .
$$

Then to prove (5.9) it is sufficient to show that

$$
\tilde{\triangle}_{L}^{Q}\left(\sigma\left(T_{\alpha}\right)\right)=4 \alpha \sigma\left(T_{\alpha}\right)
$$

Given a pair $n \geq 1$ and $m \geq 1$, one has, by direct computation

$$
\begin{aligned}
\left\langle\sigma\left(T_{\alpha}\right)^{\prime \prime}(\xi, \eta),\left(e_{n} \oplus f_{m}\right)^{\otimes 2}\right\rangle & =\left[2\left(\int_{0}^{\alpha} e_{n}^{2}(u) d u+\int_{0}^{\alpha} f_{m}^{2}(u) d u\right)\right. \\
+ & \left.4\left(\int_{0}^{\alpha} \xi(u) e_{n}(u) d u+\int_{0}^{\alpha} \eta(u) f_{m}(u) d u\right)^{2}\right] \sigma\left(T_{\alpha}\right)(\xi, \eta) .
\end{aligned}
$$

Then taking the conditions $(\mathbf{j})$ and (jj) into account, we obtain

$$
\tilde{\triangle}_{L}^{Q}\left(\sigma\left(T_{\alpha}\right)\right)(\xi, \eta)=4 \alpha \sigma\left(T_{\alpha}\right)(\xi, \eta), \quad \xi, \eta \in N
$$

as desired. The verification of the second assertion is straightforward. 
Corollary 5.4. Let $S$ be a compact interval of $\mathbb{R}$ equipped with a finite measure $\nu$. If the assumptions in Theorem 5.3 are true for all $s \in S$, then

$$
L_{t}=\int_{S} e^{2 \gamma s t} T_{s} \nu(d s), \quad t \geq 0,
$$

satisfies the quantum white noise heat equation

$$
\frac{\partial L}{\partial t}=\frac{\gamma}{2} \triangle_{L}^{Q} L
$$

with initial condition

$$
L_{0}=\int_{S} T_{s} \nu(d s)
$$

Proof. Since $s \mapsto T_{s} \in \mathcal{L}\left(\mathcal{F}_{\theta}\left(N^{\prime}\right), \mathcal{F}_{\theta}\left(N^{\prime}\right)^{*}\right)$ is continuous, $\left\{T_{s} ; s \in S\right\}$ is a compact subset of $\mathcal{L}\left(\mathcal{F}_{\theta}\left(N^{\prime}\right), \mathcal{F}_{\theta}\left(N^{\prime}\right)^{*}\right)$ and $s \mapsto e^{2 \gamma s t} T_{s}$ is then integrable, and hence $L_{t} \in$ $\mathcal{L}\left(\mathcal{F}_{\theta}\left(N^{\prime}\right), \mathcal{F}_{\theta}\left(N^{\prime}\right)^{*}\right)$. By the Lebesgue dominated convergence theorem we obtain

$$
\frac{\partial}{\partial t}\left(L_{t}\right)=\frac{\gamma}{2} \int_{S} 4 s T_{s} e^{2 \gamma s t} \nu(d s)=\frac{\gamma}{2} \int_{S} \triangle_{L}^{Q}\left(T_{s}\right) e^{2 \gamma s t} \nu(d s)=\frac{\gamma}{2} \triangle_{L}^{Q}\left(L_{t}\right)
$$

which is what we look for.

In view of Lemma 3.2 and Corollary 5.4 we obtain the following well-known result, see e.g. [22], [24].

Corollary 5.5. Let $\left\{\Phi_{s}\right\} \subset \mathcal{F}_{\theta}\left(N^{\prime}\right)^{*}$ be the classical stochastic process corresponding to the quantum stochastic process determined by (5.2), i.e., $\Phi_{s}=T_{s} e_{0}, s \in S$. Under the assumptions of Theorem 5.3, the integral

$$
\Psi_{t}=\int_{S} e^{\gamma s t} \Phi_{s} d s
$$

is defined in $\mathcal{F}_{\theta}\left(N^{\prime}\right)^{*}$ and satisfies the Lévy heat equation

$$
\frac{\partial}{\partial t} \Psi_{t}=\frac{\gamma}{2} \triangle_{L} \Psi_{t}
$$

with the initial condition

$$
\Psi_{0}=\int_{S} \Phi_{s} d s
$$

REMARK 5.6. The Lévy Laplacian [19] has been extensively studied in white noise analysis, see e.g. [14], [17], [18]. It is noteworthy that in these different developments the Lévy Laplacian is defined depending on an infinite sequence $e=\left(e_{n}\right)_{n \in \mathbb{N}}$ of the middle Hilbert space $H$ of the initial nuclear Gelfand triple $E \subset H \subset E^{\prime}$ on which is constructed the algebra of white noise operators, $\left(\mathcal{L}\left(\mathcal{F}_{\theta}\left(N^{\prime}\right), \mathcal{F}_{\theta}\left(N^{\prime}\right)^{*}\right)\right.$ in our case). A typical example, as used in the previous Section, is $E=S(\mathbb{R}) \subset H=L^{2}(\mathbb{R}, d t) \subset E^{\prime}=S^{\prime}(\mathbb{R})$. It is usually assumed that $e=\left(e_{n}\right)_{n \in \mathbb{N}}$ satisfies the following three properties:

(i) $e=\left(e_{n}\right)_{n \in \mathbb{N}}$ is a complete orthonormal basis of $L^{2}(S, d t)$ with $S$ a finite time interval.

(ii) $e=\left(e_{n}\right)_{n \in \mathbb{N}}$ is equally dense, i.e.,

$$
\lim _{n \rightarrow \infty} \frac{1}{n} \sum_{k=1}^{n} \int_{S} f(u) e_{k}^{2}(u) d u=\int_{S} f(u) d u, \quad \forall f \in L^{\infty}(S, d t) .
$$


(iii) $e=\left(e_{n}\right)_{n \in \mathbb{N}}$ is uniformly bounded on $S$, i.e.,

$$
\sup _{n \geq 0}\left\|e_{n}\right\|_{\infty}<\infty
$$

where $\|\cdot\|_{\infty}$ is the supremum norm.

Recently, more attention has been paid to the sufficiency of such conditions. Weaker assumptions on $e=\left(e_{n}\right)_{n \in \mathbb{N}}$ are first considered by Obata-Ouerdiane [24], in order to solve the heat equation with the Lévy Laplacian acting on functions on a real nuclear space $E$. More precisely, the Lévy Laplacian is defined depending on an arbitrary infinite sequence $e=\left(e_{n}\right)_{n \in \mathbb{N}}$. Moreover, it is shown that a class of solutions of the Lévy heat equation can be obtained in a unified manner without assuming the above traditional conditions (i), (ii), (iii), but just under the two conditions (j) and (jj) as in Theorem 5.3 for two variables.

Below we delve a little further into the weakness of these new hypotheses. First, note that the condition (jj) is clearly strictly weaker than (ii). On the other hand, let $e_{1}$ be a fixed nonzero vector in $E$ with $\left\|e_{1}\right\|_{L^{2}(0,1)}=1$, and for any $n \geq 1$, put $e_{n}=\frac{1}{n} e_{1}$. Then we shall focus on this sequence $e=\left(e_{n}\right)_{n \in \mathbb{N}}$. For $\xi \in E$ we have

$$
\lim _{n \rightarrow \infty} \frac{1}{n} \sum_{k=1}^{n}\left\langle e_{k}, \xi\right\rangle^{2}=\lim _{n \rightarrow \infty} \frac{1}{n} \sum_{k=1}^{n} \frac{\left\langle e_{1}, \xi\right\rangle^{2}}{k^{2}}=0 .
$$

This means that the condition $(\mathbf{j})$ is satisfied with the just constructed sequence $\left(e_{n}\right)$, but this sequence is not an orthogonal coordinate system as in (i). In conclusion, the conditions pointed out by Obata-Ouerdiane [24] are strictly weaker than the traditional ones.

\section{References}

[1] L. Accardi, H. Ouerdiane and O. G. Smolyanov, Lévy Laplacian acting on operators, Russian J. Math. Phys. 10 (2003), 359-380.

[2] L. Accardi and N. Obata, On the Fock space realization of the Lévy Brownian motion, Volterra Preprint, 1994.

[3] L. Accardi, P. Gibilisco and I. V. Volovich, Yang-Mills gauge fields as harmonic functions for the Lévy Laplacian, Russian J. Math. Phys. (1994).

[4] L. Accardi, P. Roselli and O. G. Smolyanov, The Brownian motion generated by the Lévy Laplacian, Mat. Zametki 54 (1993), 144-148.

[5] N. Asai, I. Kubo, and H.-H. Kuo, General characterization theorems and intrinsic topologies in white noise analysis, Hiroshima Math. J. 31 (2001), 299-330.

[6] M. Ben Chrouda, M. El Oued and H. Ouerdiane, Convolution calculus and applications to stochastic differential equations, Soochow Journal of Mathematics 28 (2002), 375-388.

[7] M. Ben Chrouda and H. Ouerdiane, Algebras of operators on holomorphic functions and applications, Mathematical Physics Analysis and Geometry 5 (2002), 65-76.

[8] F. A. Berezin, Wick and anti-wick operator symbols, Math. USSR Sbornik 15 (1971), 577-606.

[9] D. M. Chung, U. C. Ji and N. Obata, Quantum stochastic analysis via white noise operators in weighted Fock space, Rev. Math. Phys. 14 (2002), 241-272. 
[10] D. M. Chung, U. C. Ji and N. Obata, Higher powers of quantum white noises in terms of integral kernel operators, Infinite Dimen. Anal. Quantum Prob. 1 (1998), 533-559.

[11] S. Dineen, Complex Analysis on Infinite Dimensional Spaces, Springer-Verlag, 1999.

[12] R. Gannoun, R. Hachaichi, H. Ouerdiane and A. Rezgi, Un théorème de dualité entre espaces de fonctions holomorphes à croissance exponentielle, J. Funct. Anal. 171 (2000), $1-14$.

[13] I. M. Gelfand and N. Ya. Vilenkin, Generalized Functions, Vol. 4, Academic Press, New York and London, 1964.

[14] T. Hida, A role of the Lévy Laplacian in the causal calculus of generalized white noise functionals, in: Stochastic Processes, A Festschrift in Honour of G. Kallianpur (S. Cambanis et al., eds.) Springer-Verlag, 1992.

[15] T. Hida, H.-H. Kuo, J. Potthoff and L. Streit, White Noise: an Infinite Dimensional Calculus, Kluwer Academic, 1993.

[16] U. C. Ji, N. Obata and H. Ouerdiane, Analytic characterization of generalized Fock space operators as two-variable entire functions with growth condition, Infinite Dimensional Analysis Quantum Probability and Related Topics 5 (2002), 395-407.

[17] H.-H. Kuo, N. Obata and K. Saitô, Diagonalization of the Lévy Laplacian and related stable processes, Infinite Dimensional Analysis Quantum Probability and Related Topics 5 (2002), 317-331.

[18] H.-H. Kuo, White Noise Distrubition Theory, CRC Press, Boca Raton 1996.

[19] P. Lévy, Leçons d'analyse fonctionnelle, Gauthier-Villars, 1922.

[20] V. E. Nazaikinskii, V. E. Shatalov and B. Yu. Sternin, Methods of Noncommutative Analysis, de Gruyter, 1995.

[21] N. Obata, White Noise Calculus and Fock Spaces, Lecture Notes in Mathematics 1577, Springer-Verlag, 1994.

[22] N. Obata, Quadratic quantum white noise and Lévy Laplacian, Nonlinear Anal. 47 (2001), $2437-2448$.

[23] N. Obata, Quantum white noise calculus based on nuclear algebras of entire functions, in: Trends in Infinite Dimensional Analysis and Quantum Probability (Kyoto 2001), RIMS No. $1278,130-157$.

[24] N. Obata and H. Ouerdiane, Heat equation associated with Lévy Laplacian, in: Proc. Internat. Conf. Stochastic Analysis and Applications, S. Albeverio, A. B. de Monvel and H. Ouerdiane (eds.), Kluwer Academic Publishers, 2004, 53-68.

[25] K. Saitô, A (C $\left.C_{0}\right)$-group generated by the Lévy Laplacian, Infinite Dimensional Analysis Quantum Probability and Related Topics 1 (1998), 425-437. 
